\title{
An Indoor Flying Platform with Collision Robustness and Self-Recovery
}

\author{
Adam Klaptocz, Grégoire Boutinard-Rouelle, Adrien Briod, Jean-Christophe Zufferey, Dario Floreano
}

\begin{abstract}
This paper presents a new paradigm in the design of indoor flying robots that replaces collision avoidance with collision robustness. Indoor flying robots must operate within constrained and cluttered environments where even nature's most sophisticated flyers such as insects cannot avoid all obstacles and should thus be able to withstand collisions and recover from them autonomously. A prototype platform specifically designed to withstand collisions and recover without human intervention is presented. Its dimensions are optimized to fulfill the varying constraints of aerodynamics, robustness and self-recovery, and new construction techniques focusing on shock absorption are presented. Finally, the platform is tested both in-flight and during collisions to characterize its collision robustness and self-recovery capability.
\end{abstract}

\section{INTRODUCTION}

Navigation of indoor environments by mobile robots has many applications, such as the exploration and surveillance of collapsed buildings or radioactive areas. Flight as indoor locomotion is interesting because it is not constrained by the morphology of the ground and can be used to navigate through staircases or elevator shafts much quicker than ground-based locomotion. Indoor flying platforms however are constrained by the small cluttered environments in which they must operate. Flight within small enclosures requires small size, maneuverability, and slow speed, but most importantly local obstacle detection to remain airborne.

Most current flying robots are designed for flight outdoors in open terrain where collisions are unlikely [1], [2], though some effort has been made to include obstacle avoidance outdoors [3], [4]. Indoor collision avoidance has focused on lightweight and passive sensors and low-computation algorithms such as optic flow-based navigation [5], [6], and although having some success, these methods are far from robust and collisions remain unavoidable. In fact even nature's most successful flyers such as insects, though capable of impressive flight indoors, still frequently crash into obstacles such as windows and low-contrast walls.

Exposed blades on many current platforms result in catastrophic failures after even the smallest contact with an obstacle. Carbon rods around the platform or the rotors [7]-[9] provide some collision protection, but cannot consistently keep the platform airborne after contact. Once on the ground, most indoor flying robots cannot take off again without human intervention. Some helicopters that can land autonomously and take off again exist [10], though only if

This work was supported by the Swiss National Science Foundation (grant 200020-116149) and armasuisse, competence sector Science + Technology for the Swiss Federal Department of Defense, Civil Protection and Sports.

A. Klaptocz, A. Briod, J.-C. Zufferey and D. Floreano are with the Laboratory of Intelligent Systems, Ecole Polytechnique Fédérale de Lausanne, Lausanne, Switzerland

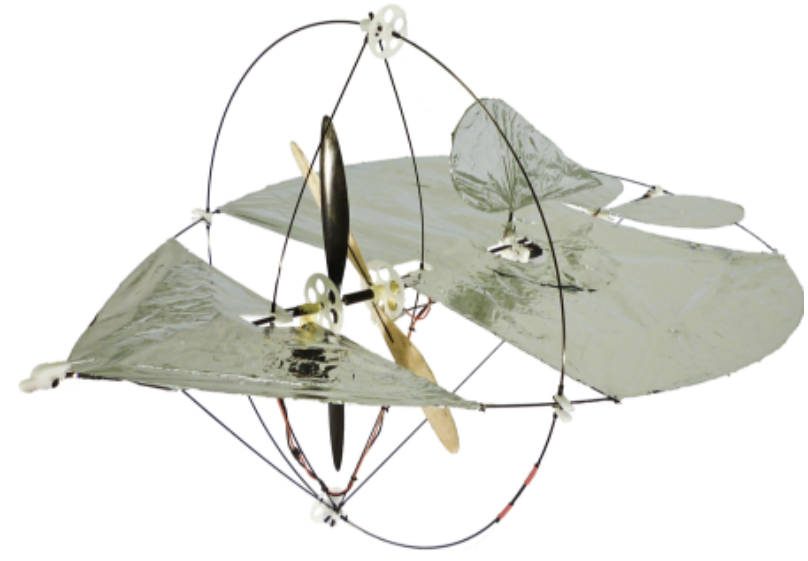

Fig. 1. The completed prototype flying platform

they land on their feet. No provisions are made for landing upside-down, or for collisions with obstacles that cause loss of flight control.

Several robots that locomote through jumping have dealt with the problem of righting themselves after falling to the ground. Most devices are passive, based on spherical cages and intelligent distribution of the Centre of Gravity (COG) [11]-[13] or a moving COG that induces rolling. These platforms however do not have to worry about aerodynamic constraints on their COG inherent to flying systems.

In this paper we propose a new paradigm in the design of indoor flying robots; instead of concentrating on avoiding collisions we designed a platform that can withstand contact with obstacles and take off again without human intervention, no matter what position the platform lands in. The paper begins by defining the requirements of robust indoor flight. The design of a novel indoor flyer will then be presented along with its self-recovery abilities. A mathematical model used to optimize the platform's shape will be shown, followed by flight tests and collision tests of the prototype platform (Fig. 1).

\section{PLATFORM DESIGN}

\section{A. Requirements}

Besides typical aerodynamic considerations that apply to all flying platforms, several additional requirements were set for the design of this new platform:

- Size: Small size is required for the platform to fit through doors and windows. A maximum dimension of $40 \mathrm{~cm}$ was chosen for this design.

- Slow forward flight: Cluttered indoor environments necessitate slow flight (in the order of 1-2 m/s) and 
maneuverability to avoid most collisions and remain airborne. Forward flight facilitates the use of optic flowbased obstacle avoidance [6].

- Hovering: The ability to hover, though not absolutely necessary, is useful in navigating constrained environments.

- Collision robustness: The platform must be able to withstand collisions at full speed with hard objects such as walls. The ability to remain airborne after light contact with objects is also beneficial.

- Autonomous self-recovery: The platform should be able to take off again after a collision that results in a fall to the ground from any possible falling position without any human intervention.

\section{B. Design Choices}

There are many types of flying vehicles, such as airships, wing-based airplanes, flapping-wing platforms, and various configurations of rotorcraft, all of which present their particular advantages and drawbacks. Airships [14] have a very poor volume-to-lift ratio and a large inertia, making them difficult to maneuver in cluttered environments. Flapping-wing platforms [15] are mechanically complex and their wings are difficult to protect in case of collisions with the environment. Compared to rotorcraft, wing-based platforms are more efficient in forward flight [16] but require a minimum forward speed to remain aloft [17]. Rotorcraft satisfy the hovering requirement but require more energy since they must create all their lift using their rotor. A hybrid design was thus chosen that integrates the efficiency in flight of a winged platform and the hovering ability of a rotorcraft.

An initial platform design was created based on a teardropshaped wing with a ring around the propellers that are placed within the wing surface near the front of the platform, as shown in Fig. 2. This simple design uses a single carbon rod for the perimeter of the wing and a second for the ring. These two rods remain flexible and absorb the shock during contact with an object or the ground, thus increasing collision robustness. The propellers and control surfaces as well as vital components such as drive electronics and sensors remain within the perimeter of these exterior carbon rods and are thus protected from collisions. Using a minimum number of carbon rods also keeps the platform lightweight and easy to build. The addition of contra-rotating propellers and a powerful drivetrain allow the platform to fly at slow speeds and to fly in a vertical position with propellers pointing upwards (prop-hang), and thus hover like a rotorcraft when required.

A passive system for autonomous self-recovery by means of the geometry of the platform and positioning of the COG was chosen for this design. Active systems such as thrust vectoring or actuated appendages (mimicking the legs used by insects to recover when they fall on their backs [18]) were considered, but their added weight, complexity and development time were deemed unnecessary for a first prototype. When the platform lands on its front (Fig. 3a) or on its side (Fig. 3b) the position of the COG rotates
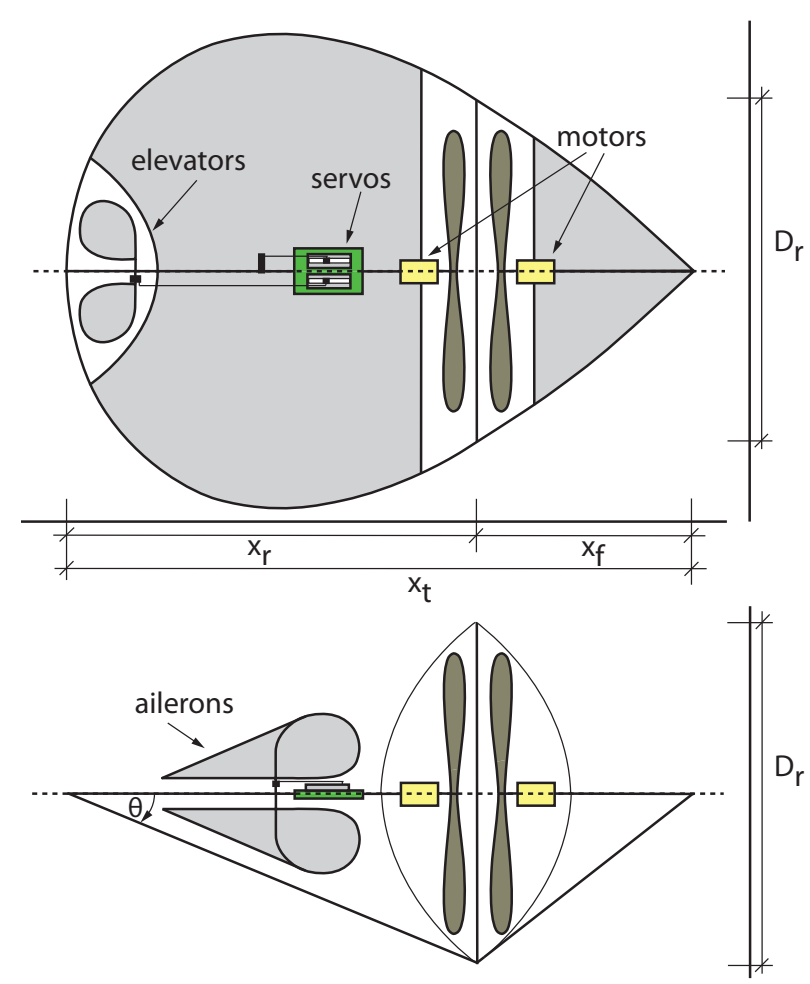

Fig. 2. Top (above) and side (below) views of the conceptual design of the platform. The teardrop shape of the wing and the takeoff angle $\theta$ are defined by the diameter $D_{r}$ and position $x_{r}$ of the ring protecting the propellers, and the total length $x_{t}$ of the platform.

the platform about the ring or the wing. The platform thus has only two stable positions when on the ground (Fig. 3c), both of which have the propeller pointing upwards, ready for takeoff.

\section{Dimensioning}

The selected design has to be dimensioned carefully to fulfill the requirements of aerodynamic stability, efficiency and self-recovery capabilities. Maximizing the surface area $A$ of the wing increases its lift, which in turn decreases required flight speed for a given weight [16]. Increasing the diameter of the propellers increases their efficiency [19] as well as the available thrust for a given motor size, required when hovering. Aerodynamic stability in forward flight is defined by the correct placement of the COG (see Fig. 4) with respect to the wing. Self-recovery also constrains the position of the COG to allow passive recovery. In addition the takeoff angle $\theta$ should be maximized to facilitate takeoff.

All of these parameters except for the position of the COG are defined by the geometry of the platform, which in turn is defined by three geometric parameters: the diameter of the ring $D_{r}$, its position $x_{r}$ along the main axis of the platform, and the total length $x_{t}$ of the platform (see Fig. 2). A length $x_{t}$ of $40 \mathrm{~cm}$ was chosen to remain within the size constraints of indoor flight, thus there remain only two dimensions that can be optimized to find a suitable platform shape.

The COG of the platform is an important constraint, as it must be placed to allow both upturning when on the ground 


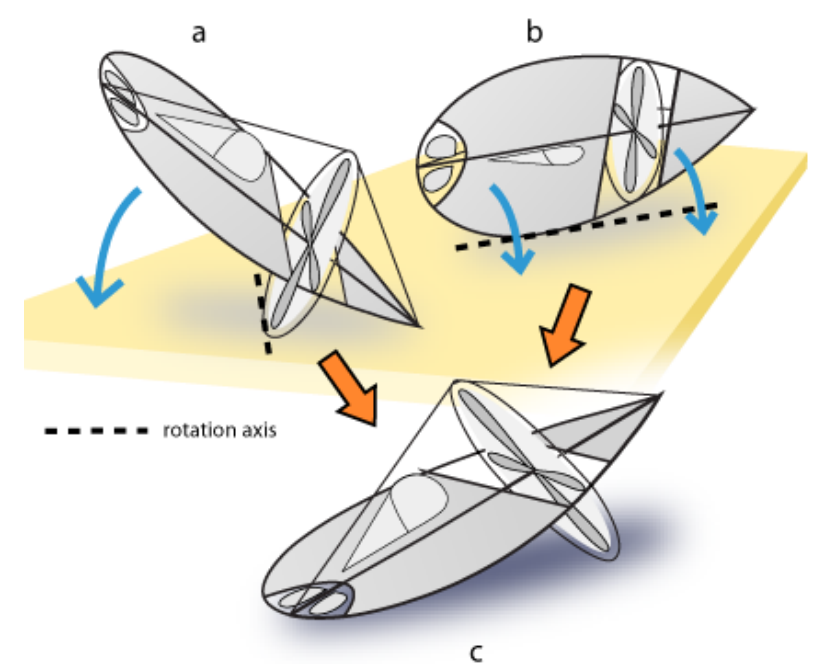

Fig. 3. Two possible unstable landing positions. When landing nose-first (a), the weight of the back of the platform pivots it around the ring into a stable position (c). When landing on the side of the wing (b), the platform can roll to either side, again settling into a stable position (c). Only one stable position is shown (c), the other one being symmetrical but upsidedown.

and aerodynamic stability while in flight (see Fig. 4). These two constraints must therefore be evaluated to ensure that a position exists in which they overlap. It is assumed that the COG can be placed in any position during construction by strategically displacing heavy components such as the battery or the electronics.

The first step is the dimensioning of the wing. Bending a thin carbon rod into a circle and then connecting the two ends at any desired angle results in a teardrop shape that can be closely approximated by the following equation:

$$
y=\sin (\arccos (x)) \sin ^{m}\left(\frac{\arccos (x)}{2}\right)
$$

The parameter $m$ defines the shape of the wing (see Fig. 5), and can be calculated by solving Eq. 1 at the point $x=$ $x_{r}, y=\frac{D_{r}}{2}$, defined by the position and size of the ring. Once the parameter $m$ is known, the total surface area of the wing can be calculated by integrating Eq. 1 from $x=-1$ to $x=1$, while taking into account the space required for the propellers.

The takeoff angle $\theta$ of the platform at rest (shown in Fig. 2) can be calculated geometrically using the formula:

$$
\theta=\arctan \left(\frac{D_{r}}{2 x_{r}}\right)
$$

The position of the COG for self-recovery from a nosedown position, $C_{u}$, must be placed behind the pivot point $O$ on the ring (see Fig. 4). The force of gravity acting on the COG then creates a moment that rotates the platform into takeoff position. It can be calculated as follows:

$$
C_{u}<x_{u}, x_{u}=x_{t}-\left(\frac{D_{r}^{2}}{4 x_{f}}+x_{f}\right)
$$

where $C_{u}$ is the COG required for self-recovery and $x_{u}$ is the minimum distance of $C_{u}$ from the front of the platform.

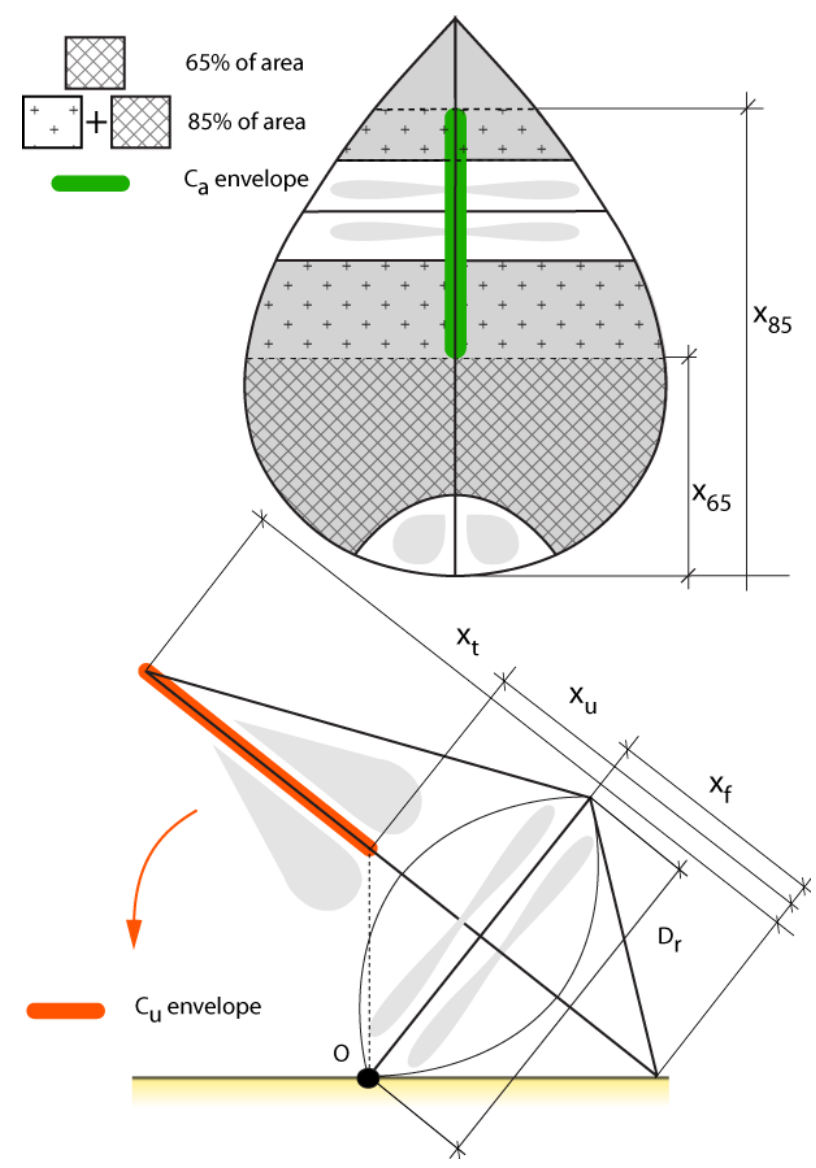

Fig. 4. Aerodynamic constraints (above, top view of platform) require the COG to be placed between $65 \%$ and $85 \%$ of the wing surface area; this defines the $C_{a}$ envelope. Self-recovery (below, side view of platform) requires the COG to be placed behind the point perpendicular to the pivot point $O$; this defines the $C_{u}$ envelope. The COG must be within both the $C_{a}$ and the $C_{u}$ envelopes to ensure flight and self-recovery.

The COG position allowing aerodynamically-stable flight $C_{a}$ is more difficult to calculate. It must be far enough forward to prevent stalling yet far enough back to prevent diving. Formulas exist for many standard shapes of wing and aerofoil. For example, a straight wing in a classical plane with a tail should have its COG at $1 / 4$ the chord distance from the leading edge [17]. However, the dual wings, flat yet flexible wing material and teardrop shape of the proposed design are all unconventional. Experiments were thus conducted using a mockup wing with a $10 \mathrm{~g}$ weight placed in varying positions along the wing's main axis to simulate the weight of the platform. The performance of the wing was evaluated through repeated trials to determine the COG positions that yield aerodynamically stable flight. Based on flight tests with the mockup, the value of $C_{a}$ can be conservatively estimated using the equation:

$$
x_{65}>C_{a}>x_{85}
$$

where $x_{65}$ and $x_{85}$ are the points behind which $65 \%$ and $85 \%$ of the total surface area of both the front and the back parts of the wing reside, respectively (see Fig. 4). 


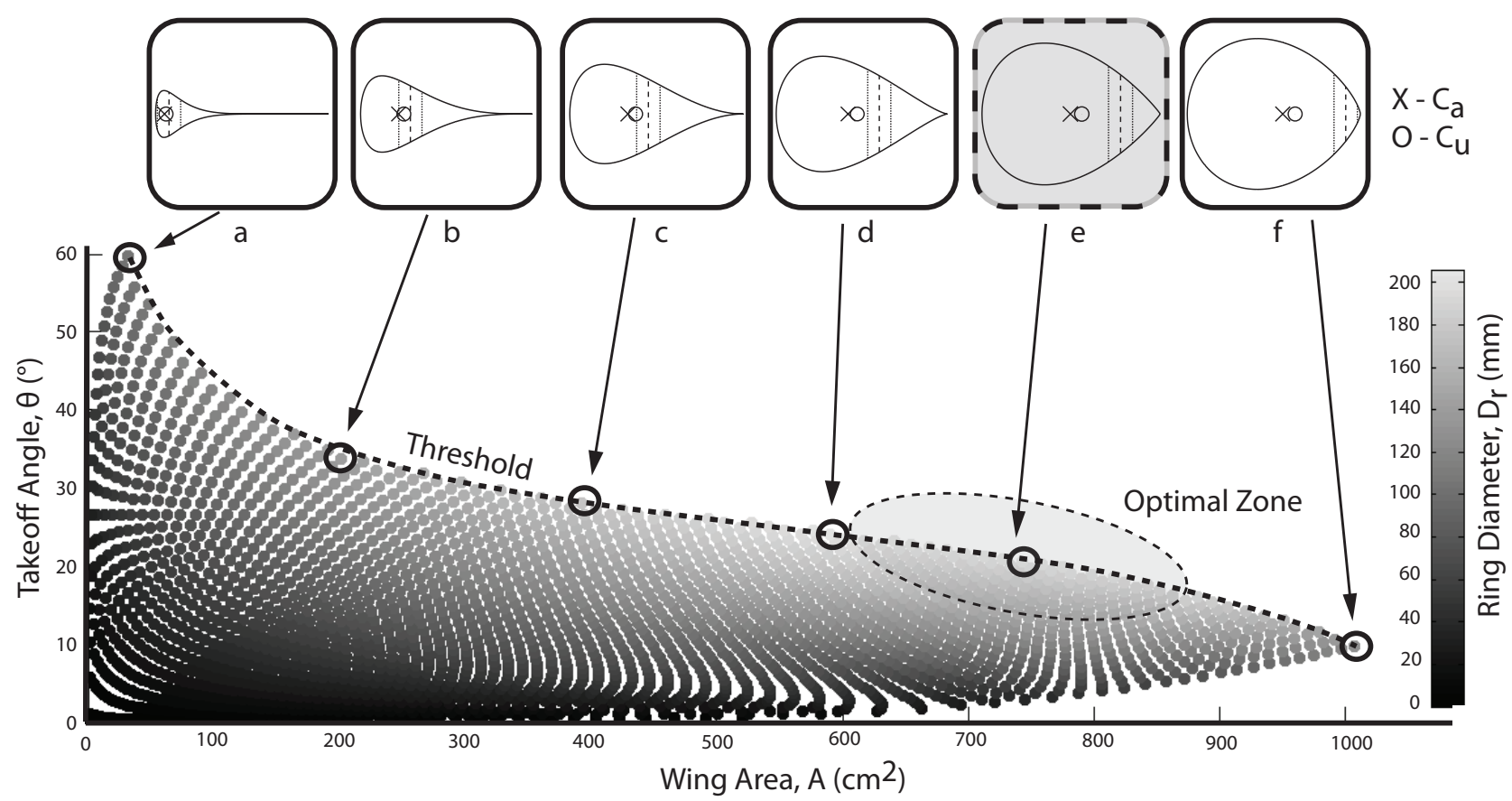

Fig. 6. To maximize takeoff angle, wing surface area and ring diameter various configurations of these parameters for a platform length $x_{t}$ of $40 \mathrm{~cm}$ are presented. All points below the threshold line represent configurations at which the COG requirements of flight and self-recovery are fulfilled. An optimal zone exists where both the wing area and ring diameter are maximized without greatly affecting the takeoff angle. Above the graph are six sample configurations that aid to visualize the parameters corresponding to the threshold line, with $C_{a}$ represented by an $\mathrm{X}$ and $C_{u}$ represented by a circle. Although all these configurations meet the COG requirements in theory, some are impractical to implement in a physical platform (such as the examples $\mathrm{a}$ and $\mathrm{b}$ due to their small wing area and $\mathrm{f}$ due to its small ring size). Configuration $\mathrm{e}$ is the one that was selected for the prototype flying platform.

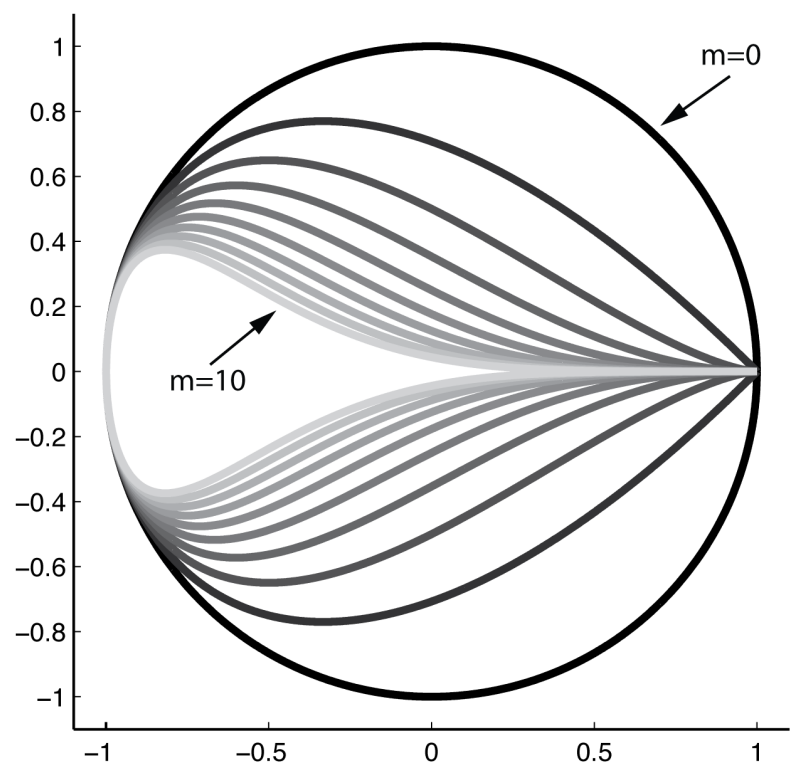

Fig. 5. The Teardrop curves, the natural shape taken by a single carbon fibre rod with its ends attached, for $m=0$ (circle) to $m=10$.

\section{PROTOTYPE REALIZATION}

The takeoff angle $\theta$, wing surface area $A$ and COG envelopes $C_{a}$ and $C_{u}$ were calculated for a series of ring positions $x_{r}$ and diameters $D_{r}$ ranging from 1 to $40 \mathrm{~cm}$ and with a fixed platform length $x_{t}$ of $40 \mathrm{~cm} . C_{a}$ and $C_{u}$ were computed for each configuration, and only those that fulfilled both requirements were considered valid. The main parameters to maximize are the wing area, takeoff angle and the size of the ring, which defines the diameter of the propellers.

Fig. 6 presents the range of possible configurations that satisfy the requirements of COG positioning. Though configurations exist with takeoff angles of up to $60^{\circ}$, they do not have sufficient wing area for flight. Considering a minimum wing area of $200 \mathrm{~cm}^{2}$, practical solutions begin with a takeoff angle of less than $30^{\circ}$. This angle diminishes by only $10^{\circ}$ for wing areas ranging from 300 to over $800 \mathrm{~cm}^{2}$, and thus the area can be maximized without greatly affecting the takeoff angle. The ring size also increases with the wing area, though it reaches a maximum at a wing area of around $750 \mathrm{~cm}^{2}$.

There is no single architecture that maximizes all required parameters, though there is a certain optimal zone in which wing area and ring diameter can be maximized without greatly affecting the takeoff angle. A design within this optimal zone was chosen, with the maximum ring size of $206 \mathrm{~mm}$, a wing area of $759 \mathrm{~cm}^{2}$ and takeoff angle of $20.1^{\circ}$, which corresponds to a ring position of $281 \mathrm{~mm}$ from the back of the platform. This configuration has $C_{a}$ and $C_{u}$ positions of $186.5 \mathrm{~mm}$ and $191.85 \mathrm{~mm}$, respectively, thus there exists a margin of $5 \mathrm{~mm}$ to position the COG to allow both aerodynamic stability and upturning.

A prototype flying platform was built using the abovementioned optimized values (see Fig. 7). Thrust was provided 


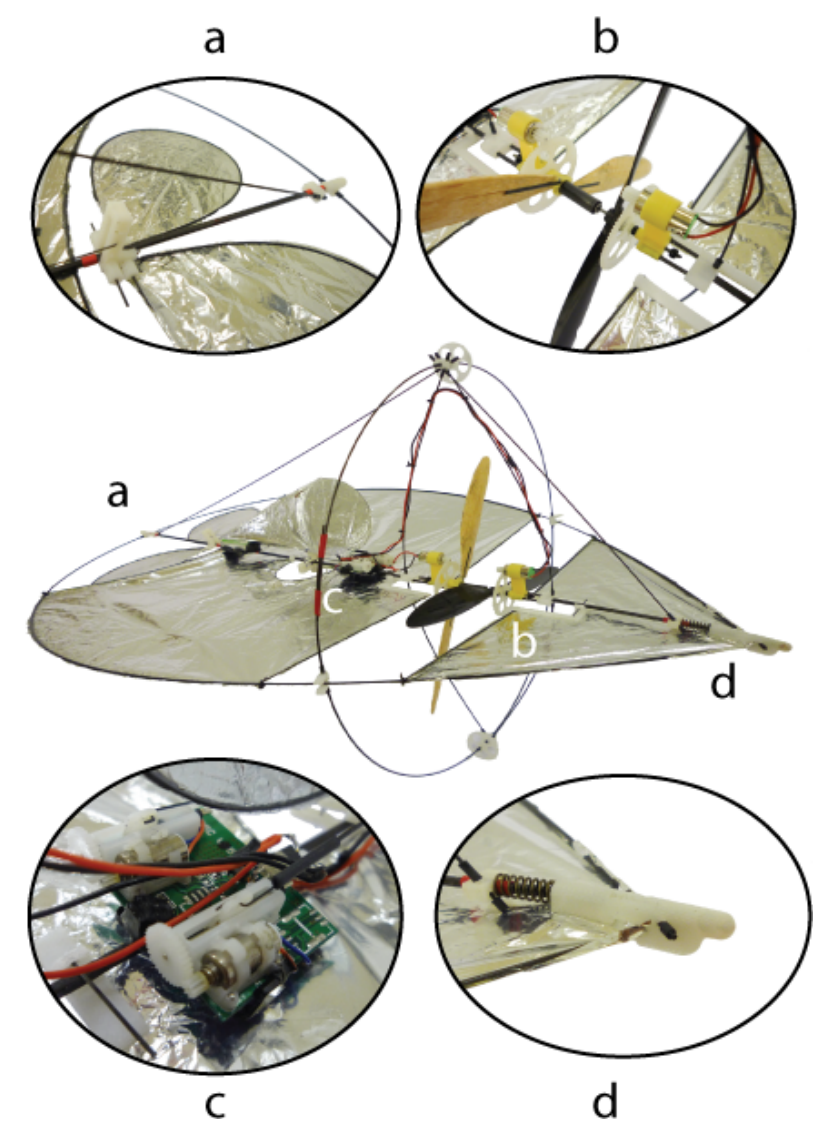

Fig. 7. The prototype flying platform with details of various subsystems. (a) depicts the linked dual elevator assembly and the connector between the wing and the back of the main bar, both printed using a 3D printer. (b) shows the coaxial motor assembly, linked using miniature ball bearings. (c) is the off-the-shelf motor-control board that features two on-board linear servos. (d) details the spring mechanism for absorbing frontal impacts and separating the wing from the main axis of the prototype.

by two $6 \mathrm{~mm}$ DC motors with $14 \mathrm{~mm}$ contra-rotating propellers placed within the ring, providing a total of $30 \mathrm{~g}$ of static thrust. Energy is provided by a $110 \mathrm{mAh}$ lithiumpolymer battery. The total weight of the platform is of $20.5 \mathrm{~g}$. The platform is controlled through two miniature servomotors actuating a rudder and an elevator, which regulate altitude and yaw in forward flight as well as in hover. Though no control of roll was implemented for hover mode due to limitations of the off-the-shelf electronics, this could be implemented through individual control of the propellers or the use of an additional servo and differential actuation of the two elevators.

Instead of using glue, miniature mechanical pieces printed using a 3D printer assured the connections between the various carbon rods and components of the structure. In addition to easing construction, the pieces allowed most of the carbon rods to be replaced in case of a failure without the need to rebuild the entire structure. The same pieces can also be used to build platforms with varying dimensions with minimal effort.

Another innovative feature is the separation of the main wing from the rest of the platform (see Fig. 7). This allows the wing to flex during a head-on collision and absorb the shock while the main bar of the structure remains decoupled through a spring, which absorbs additional energy (Fig. 7d). The wing is fixed to the rest of the platform at the back (Fig. 7a), while the rest of the connection points are allowed to slide along the main bar.

\section{RESULTS}

The prototype was put through remote-controlled flight tests in a $6 \times 7 \mathrm{~m}$ experimentation room and proved an agile flyer both in forward flight and in hover. Transition between hover and forward flight, and vice versa, was smooth and easily controllable, partly due to the backward placement of the COG. The platform can fly for approximately $10 \mathrm{~min}$ with a fully-charged $110 \mathrm{mAh}$ battery, while spending some time in hover and some time in forward flight. The accompanying video presents the flight characteristics of the platform in both forward and hover mode ${ }^{1}$.

During flight tests the prototype had numerous collisions with objects or surfaces such as walls or the ceiling. These collisions provided several insights into the self-recovery capabilities of the platform:

- Light contact with walls did not always cause the platform to fall to the ground. It could in fact fly along the wall, its front tip grazing the surface. This behavior resembles insects flying against a window pane looking for an exit.

- After collisions with an object that cause a fall to the ground, the prototype always settled to one of two stable positions on the ground, and in most cases could take off again without human intervention.

To further test the platform's resilience to collisions, the platform was systematically dropped from a height of $1 \mathrm{~m}$ from a variety of different starting positions. Highspeed video was taken of each collision to try to analyze the deformation of the structure during a collision. As the platform hits the ground, the shock is partially absorbed by the spring at the nose of the platform (Fig. 7d), and partially by the deformation of the wing. Fig. 8 shows frames from a typical collision and subsequent righting of the platform after a head-on collision with the ground. This collision can be seen in more detail in the attached video.

\section{CONCLUSION}

This paper presents a paradigm shift in the design of practical indoor flying platforms that replaces collision avoidance with collision robustness. A prototype platform capable of not only indoor flight, but of withstanding collisions and returning to the air was designed, optimized and built. Flight tests proved its ability to fly within constrained environments and to continue flying after repeated collisions with objects in the environment.

A passive self-recovery system greatly constrains the position of the COG, making it difficult to fulfill the aerodynamic

\footnotetext{
${ }^{1}$ also downloadable from http://lis.epfl.ch/airburr
} 

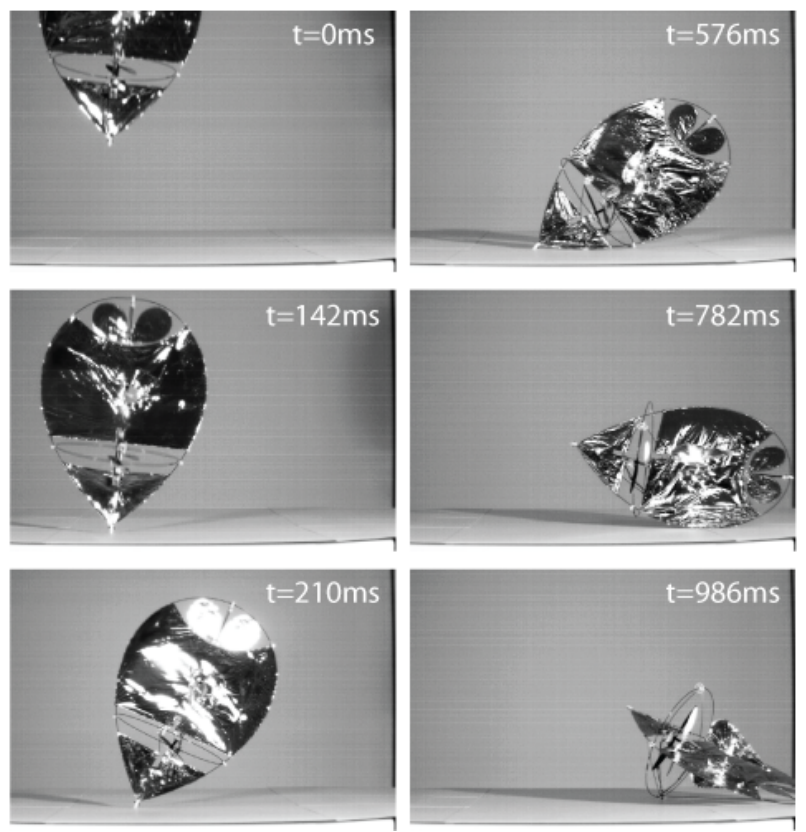

Fig. 8. Time-sequence of a typical head-on collision with the ground and subsequent self-recovery, taken with a high-speed camera. The platform rolls onto its side before rolling into takeoff position.

constraints required for flight and limiting the possible platform geometries. Though able to self-recover autonomously in many situations, there are still many real-life scenarios that remain a challenge to the the platform, such as landing against walls or objects, in rough and uneven terrain or underneath tables or chairs. The simple passive mechanism is thus not yet sufficient for autonomous self-recovery in all cases.

Indeed, this work seems to have reached the limits of passive gravity-based recovery, and an active self-recovery system will have to be developed, along with intelligent sensing and control to detect the current flight or ground position, detect collisions and their severity and to take off autonomously. These future steps will bring our platform closer to the agile indoor flight that resembles more closely the amazing capabilities of the insects that fly through our homes.

\section{ACKNOWLEDGMENTS}

This work was supported by the Swiss National Science Foundation (grant 200020-116149) and armasuisse, competence sector Science + Technology for the Swiss Federal Department of Defense, Civil Protection and Sports.

\section{REFERENCES}

[1] J. Grasmeyer and M. Keennon, "Development of the black widow micro air vehicle," in Fixed and Flapping Wing Aerodynamics for Micro Air Vehicle Applications, ser. Progress in Astronautics and Aeronautics, T. J. Mueller, Ed. AIAA, 2001, vol. 195, pp. 519-535.

[2] F. Caballero, L. Merino, J. Ferruz, and A. Ollero, "Vision-based odometry and slam for medium and high altitude flying uavs," Journal of Intelligent and Robotic Systems, vol. 54, no. 1-3, pp. 137-161, 2009.

[3] S. Griffiths, J. Saunders, A. Curtis, T. McLain, and R. Beard, "Obstacle and terrain avoidance for miniature aerial vehicles," in Advances in Unmanned Aerial Vehicles: State of the Art and the Road to Autonomy, K. Valavanis, Ed. Springer, 2007, vol. 33, ch. I.7, pp. 213-244.

[4] A. Beyeler, J.-C. Zufferey, and D. Floreano, "Vision-based control of near-obstacle flight," Autonomous Robots, vol. 27, no. 3, pp. 201-219, 2009.

[5] J. How, J. Teo, and B. Michini, "Adaptive flight control experiments using raven," in 14th Yale Workshop on Adaptive and Learning Systems, Proceedings of the, 2008, pp. 205-210.

[6] J.-C. Zufferey, A. Klaptocz, A. Beyeler, J.-D. Nicoud, and D. Floreano, "A 10-gram vision-based flying robot," Advanced Robotics, Journal of the Robotics Society of Japan, vol. 21, no. 14, pp. 1671-1684, 2007.

[7] J. F. Roberts, T. Stirling, J.-C. Zufferey, and D. Floreano, "Quadrotor using minimal sensing for autonomous indoor flight," in European Micro Air Vehicle Conference and Flight Competition (EMAV2007), 2007.

[8] P. Oh, M. Joyce, and J. Gallagher, "Designing an aerial robot for hover-and-stare surveillance," in Advanced Robotics, 2005. ICAR'05. Proceedings., 12th International Conference on. IEEE, 2005, pp. 303-308.

[9] D. Schafroth, S. Bouabdallah, C. Bermes, and R. Siegwart, "From the test benches to the first prototype of the mufly micro helicopter," Journal of Intelligent and Robotic Systems, 2008.

[10] S. Saripalli, J. Montgomery, and G. Sukhatme, "Vision-based autonomous landing of an unmanned aerial vehicle," in Robotics and Automation, IEEE International Conference on, vol. 3, 2002.

[11] Y. Sugiyama, M. Yamanaka, and S. Hirai, "Circular/spherical robots for crawling and jumping," in IEEE International Conference on Robotics and Automation, 2005, pp. 3595-3600.

[12] E. Beyer and M. Costello, "Performance of a hopping rotochute," in 34th European Rotorcraft Forum, 2008.

[13] R. H. Armour and J. F. V. Vincent, "Rolling in nature and robotics: A review," Journal of Bionic Engineering, vol. 3, no. 4, pp. 195-208, 2006.

[14] J. Zufferey, A. Guanella, A. Beyeler, and D. Floreano, "Flying over the reality gap: From simulated to real indoor airships," Autonomous Robots, vol. 21, no. 3, pp. 243-254, 2006.

[15] D. Lentink, S. Jongerius, and N. Bradshaw, "The scalable design of flapping micro-air vehicles inspired by insect flight," in Flying Insects and Robots, D. Floreano, J. Zufferey, M. Srinivasan, and C. Ellington, Eds. Springer, 2009, ch. 14, in press.

[16] H. Tennekes, The Simple Science of Flight. MIT Press, 1997.

[17] M. Simons, Model Aircraft Aeordynamics. Argus Books Ltd, 1987.

[18] L. Frantsevich, "Righting kinematics in beetles (insecta: Coleoptera)," Arthropod Structure and Development, vol. 33, no. 3, pp. 221-235, 2004.

[19] J.-D. Nicoud and J.-C. Zufferey, "Toward indoor flying robots," IEEE/RSJ International Conference on Robots and Systems (IROS'02), Lausanne, pp. 787-792, 2002. 